\title{
A NOTE ON THE STRONG SUMMABILITY OF THE RIESZ MEANS OF MULTIPLE FOURIER SERIES
}

\author{
SHIGEHIKO KURATSUBO
}

ABSTRACT. Let $S_{t}^{\alpha}(x, f)$ be the Riesz means of order $\alpha$ of an integrable function $f(x)$ on $N$-dimensional torus $T^{N}(N \geqslant 2)$, that is,

$$
S_{t}^{\alpha}(x, f)=\sum_{|m|^{2}<t}\left(1-\frac{|m|^{2}}{t}\right)^{\alpha} \hat{f}(m) e^{2 \pi i m x} .
$$

E. M. Stein has shown that if $1<p \leqslant 2$ and $\alpha>\alpha_{p}$ where

$$
\alpha_{p}=\frac{N-1}{2}\left(\frac{2}{p}-1\right)-\frac{1}{p^{\prime}}=\frac{N-1}{2}-\frac{N}{p^{\prime}},
$$

then for any function $f(x) \in L^{p}\left(T^{N}\right) S_{t}^{\alpha}(x, f)$ is strong summable to $f(x)$, that is,

$$
\lim _{T \rightarrow \infty} \frac{1}{T} \int_{0}^{T}\left|S_{t}^{\alpha}(x, f)-f(x)\right|^{2} d t=0
$$

for almost every $x$. In this paper we shall show that if $1 \leqslant p \leqslant 2$ and $-1<\alpha<\alpha_{p}$, then there exists a function $f(x) \in L^{p}\left(T^{N}\right)$ such that

$$
\frac{1}{T} \int_{0}^{T}\left|S_{t}^{\alpha}(x, f)\right|^{2} d t=\Omega\left(T^{\alpha_{p}-\alpha} \log ^{-2 \tau} T\right) \quad \text { as } T \rightarrow \infty
$$

for every $x$ and every $\tau>1 / p$, in particular,

$$
\varlimsup_{T \rightarrow \infty} \frac{1}{T} \int_{0}^{T}\left|S_{t}^{\alpha}(x, f)\right|^{2} d t=\infty
$$

for every $x$, where we can take for $f(x), f_{\sigma \tau}(x)$ such that $\hat{f}_{\sigma \tau}(m)=1 /|m|^{\sigma} \log ^{\tau}|m|$, $|m|>1$.

1. Introduction. Let $R^{N}$ denote the $N$-dimensional Euclidean space, $T^{N}$ the $N$-dimensional torus (identified with the cube $Q^{N}=\left\{x=\left(x_{1}, \ldots, x_{N}\right) \in R^{N}:-\frac{1}{2}\right.$ $\left.\left.\leqslant x_{j}<\frac{1}{2}, j=1, \ldots, N\right\}\right)$ and $Z^{N}$ the integral lattice of $R^{N}$. Throughout this paper assume $N \geqslant 2$ and $1 \leqslant p \leqslant 2$.

For any $f(x) \in L^{1}\left(T^{N}\right)$, define the Riesz means of order $\alpha$ of $f(x)$ by

$$
S_{t}^{\alpha}(x, f)=\sum_{|m|^{2}<t}\left(1-\frac{|m|^{2}}{t}\right)^{\alpha} \hat{f}(m) e^{2 \pi i m x}
$$

with $\hat{f}(m)=\int_{T^{N}} f(x) e^{-2 \pi i m x} d x, m \in Z^{N}$.

Received by the editors October 26, 1984.

1980 Mathematics Subject Classification. Primary 42B05.

Key words and phrases. Multiple Fourier series, Riesz means, strong summability, $L^{p}\left(T^{N}\right)$, lattice point problem. 
The problem of the strong summability of the Riesz means of multiple Fourier series is one of dealing with the validity of the following:

$$
\lim _{T \rightarrow \infty} \frac{1}{T} \int_{0}^{T}\left|S_{t}^{\alpha}(x, f)-f(x)\right|^{2} d t=0
$$

E. M. Stein [6] has shown that if $f \in L^{p}\left(T^{N}\right)$ and $\alpha>\alpha_{p}$ where

$$
\alpha_{p}=\frac{N-1}{2}\left(\frac{2}{p}-1\right)-\frac{1}{p^{\prime}}=\frac{N-1}{2}-\frac{N}{p^{\prime}},
$$

then $\lim _{T \rightarrow \infty}(1 / T) \int_{0}^{T}\left|S_{t}^{\alpha}(x, f)-f(x)\right|^{2} d t=0$ for almost every $x$. On the other hand, it seems to us as if the case $\alpha \leqslant \alpha_{p}$ is unknown except the case $p=1$ and $\alpha=\alpha_{p}$. (In [6] Stein has stated the affirmative result without the proof.)

The purpose of this paper is to prove a negative result for the case $\alpha<\alpha_{p}$. The method consists of joining multiple Fourier series and the Lattice Point Problem in analytic number theory by means of a special function $f_{\sigma \tau}(x)$ whose Fourier coefficient is given by $\hat{f}_{\sigma \tau}(m)=1 /|m|^{\sigma} \log ^{\tau}|m|$. Our main result is the following theorem.

TheOREM. Suppose $1 \leqslant p \leqslant 2$ and $-1<\alpha<\alpha_{p}$. Then there exists a function $f(x) \in L^{p}\left(T^{N}\right)$ such that

$$
\frac{1}{T} \int_{0}^{T}\left|S_{t}^{\alpha}(x, f)\right|^{2} d t=\Omega\left(T^{\alpha_{p}-\alpha} \log ^{-2 \tau} T\right) \quad \text { as } T \rightarrow \infty
$$

for every $x$ and every $\tau>1 / p$, in particular,

$$
\varlimsup_{T \rightarrow \infty} \frac{1}{T} \int_{0}^{T}\left|S_{t}^{\alpha}(x, f)\right|^{2} d t=\infty
$$

for every $x$, where we can take $f_{\sigma \tau}(x)$ for such a function $f(x)$.

In the above statement, $g(T)=\Omega(h(T))$ implies $g(T) \neq o(h(T))$.

From this theorem we have directly the following corollary.

COROllary. Suppose $1 \leqslant p \leqslant 2$ and $0 \leqslant \alpha<\alpha_{p}$. Then there exists a function $f(x) \in L^{p}\left(T^{N}\right)$ such that

$$
S_{t}^{\alpha}(x, f)=\Omega\left(t^{(1 / 2)\left(\alpha_{p}-\alpha\right)} \log ^{-\tau} t\right) \quad \text { as } t \rightarrow \infty
$$

for every $x$ and every $\tau>1 / p$, in particular,

$$
\varlimsup_{T \rightarrow \infty}\left|S_{t}^{\alpha}(x, f)\right|=\infty
$$

for every $x$, where we can take $f_{\sigma \tau}(x)$ for such a function $f(x)$.

REMARK. The existence of $f(x) \in L^{p}\left(T^{N}\right)$ such that $\overline{\lim }_{t \rightarrow \infty}\left|S_{t}^{\alpha}(x, f)\right|=\infty$ for almost every $x$ and the fact we can take $f_{\sigma}=f_{\sigma 0}$ for such a function $f$ have been shown for $\alpha=0$ by Stein and Weiss [7] and for $0 \leqslant \alpha<(N-1) / 2$ by Babenko [3] (see also Alimov, Il'in and Nikishin [2]). Using our method, it is easy to show that their exceptional sets are empty (see also Kuratsubo [4]). 
2. Lemmas. The proof of the theorem depends on several results from $[1,5]$. These are stated here for convenience of the description. The next lemma was proved in [1, Lemma 2.2] for the case $s>0$ and $\tau=0$.

LEMMA 1. Suppose $s>-1$ and $s=r+\kappa$ where $r$ is an integer and $\kappa$ satisfies $0<\kappa \leqslant 1$. For $\beta>0$ and a nonnegative number $\tau$, define a function $b(\lambda)$ as $\lambda^{\beta} \log ^{\tau} \lambda, 0$ according to $\lambda \geqslant e, \lambda<e$ respectively. Further, for any numerical series $\sum_{k=1}^{\infty} a_{k}$, let $\sigma_{\lambda}^{s}$, $\sigma_{\lambda}^{s}$ be $\sum_{k<\lambda}(1-k / \lambda)^{s} a_{k}, \sum_{k<\lambda}(1-k / \lambda)^{s} b(k) a_{k}$ respectively. Then we have

$$
\overline{\sigma_{\lambda}^{s}}=b(\lambda) \sigma_{\lambda}^{s}+(-1)^{r+1} \int_{0}^{1} \frac{t^{r+1}}{(r+1) !} \sigma_{t \lambda}^{r+1}\left(\frac{d}{d t}\right)^{r+2}\left[(b(t \lambda)-b(\lambda))(1-t)^{s}\right] d t
$$

and, for some positive constant $C$,

$$
\int_{0}^{1} \frac{t^{r+1}}{(r+1) !}\left|\left(\frac{d}{d t}\right)^{r+2}\left[(b(t \lambda)-b(\lambda))(1-t)^{s}\right]\right| d t \leqslant C b(\lambda), \quad \lambda \geqslant 0 .
$$

Proof. The first equality is proved in [1, Lemma 2.2]. On the other hand, the second inequality follows from the next.

$$
\begin{aligned}
& \left|\left(\frac{d}{d t}\right)^{r+2}\left[(b(t \lambda)-b(\lambda))(1-t)^{s}\right]\right| \\
& \quad \leqslant C b(\lambda)\left\{\left(1-t^{\beta}\right)(1-t)^{s-r-2}+\sum_{j=1}^{r+2} t^{\beta-j}(1-t)^{s-r+j-2}\right\}
\end{aligned}
$$

and

$$
\begin{aligned}
& \int_{0}^{1} t^{r+1}\left(1-t^{\beta}\right)(1-t)^{s-r-2} d t<+\infty, \\
& \int_{0}^{1} t^{r+1+\beta-j}(1-t)^{s-r+j-2} d t<+\infty \quad(1 \leqslant j \leqslant r+2) .
\end{aligned}
$$

LEMMA 2. Under the same notation as Lemma 1, there exists a positive constant $C$ such that

$$
\frac{1}{T} \int_{0}^{T}\left|\overline{\sigma_{\lambda}^{s}}\right|^{2} d \lambda \leqslant C b(T)^{2} \sup _{0<t \leqslant T}\left(\frac{1}{t} \int_{0}^{t}\left|\sigma_{\lambda}^{s}\right|^{2} d \lambda\right), \quad T>0 .
$$

Proof. From Lemma 1, it follows that

$$
\begin{aligned}
\int_{0}^{T}\left|\overline{\sigma_{\lambda}^{s}}\right|^{2} d \lambda \leqslant & 2\left\{\int_{0}^{T} b(\lambda)^{2}\left|\sigma_{\lambda}^{s}\right|^{2} d \lambda\right. \\
& \left.+\int_{0}^{T}\left|\int_{0}^{1} \frac{t^{r+1}}{(r+1) !} \sigma_{t \lambda}^{r+1}\left(\frac{d}{d t}\right)^{r+2}\left[(b(t \lambda)-b(\lambda))(1-t)^{s}\right] d t\right|^{2} d \lambda\right\} \\
= & 2\left\{I_{1}+I_{2}\right\} .
\end{aligned}
$$


First, by monotone increasing of $b(\lambda)$ we have $I_{1} \leqslant b(T)^{2} \int_{0}^{T}\left|\sigma_{\lambda}^{s}\right|^{2} d \lambda$. Next, by Schwarz's inequality, Fubini's theorem, Lemma 1 and its proof we have

$$
\begin{aligned}
I_{2} \leqslant & \int_{0}^{T}\left(\int_{0}^{1} \frac{t^{r+1}}{(r+1) !}\left|\left(\frac{d}{d t}\right)^{r+2}\left[(b(t \lambda)-b(\lambda))(1-t)^{s}\right]\right| d t\right) \\
& \times\left(\int_{0}^{1} \frac{t^{r+1}}{(r+1) !}\left|\sigma_{t \lambda}^{r+1}\right|^{2}\left|\left(\frac{d}{d t}\right)^{r+2}\left[(b(t \lambda)-b(\lambda))(1-t)^{s}\right]\right| d t\right) d \lambda \\
& \leqslant C \int_{0}^{T} b(\lambda)^{2}\left(\int _ { 0 } ^ { 1 } t ^ { r + 1 } \left\{\left(1-t^{\beta}\right)(1-t)^{s-r-2}\right.\right. \\
& \leqslant C T b(T)^{2} \int_{0}^{1} t^{r+1}\left\{\left(1-\sum_{j=1}^{r+2} t^{\beta-j}(1-t)^{s-r+j-2}\right\}\left|\sigma_{t \lambda}^{r+1}\right|^{2} d t\right) d \lambda \\
& \leqslant C T b(T)^{2} \sup _{0<t \leqslant T}\left(\frac{1}{t} \int_{0}^{t}\left|\sigma_{\lambda}^{r+1}\right|^{2} d \lambda\right)
\end{aligned}
$$

for a suitable constant $C$, not necessarily the same at each occurrence. On the other hand, when $r<s<r+1$, let $\delta$ be a positive number such that $r+1=s+\delta$. Then we have the following well-known relation

$$
\sigma_{\lambda}^{s+\delta}=B(\delta, s+1)^{-1} \int_{0}^{1}(1-t)^{\delta-1} t^{s} \sigma_{t \lambda}^{s} d t
$$

with the beta function $B$ (e.g. [7, p. 269]). By the same calculation with $I_{2}$-estimation, we have

$$
\begin{aligned}
\frac{1}{t} \int_{0}^{t}\left|\sigma_{\lambda}^{r+1}\right|^{2} d \lambda & \leqslant B(\delta, s+1)^{-1} \int_{0}^{1}(1-u)^{\delta-1} u^{s}\left(\int_{0}^{t}\left|\sigma_{u \lambda}^{s}\right|^{2} d \lambda\right) d u \\
& \leqslant \sup _{0<u \leqslant t}\left(\frac{1}{u} \int_{0}^{u}\left|\sigma_{\lambda}^{s}\right|^{2} d \lambda\right) .
\end{aligned}
$$

These complete the proof of Lemma 2.

The next lemma is a metrical theorem of the Lattice Point Problem [5, Theorem 1] in the case $\alpha \geqslant 0$, but an examination of the proof shows that it applies without significant change to the present situation.

Lemma 3. Suppose $\alpha>-1$. Then we have

$$
\frac{1}{T} \int_{0}^{T}\left|\sum_{|m|^{2}<t}\left(1-\frac{|m|^{2}}{t}\right)^{\alpha} e^{2 \pi i m x}\right|^{2} d t=\Omega\left(T^{(N-1) / 2-\alpha}\right)
$$

for every $x$. 
Applying Lemma 2 to the case

$$
a_{k}=\sum_{|m|^{2}=k} \frac{e^{2 \pi i m x}}{|m|^{\sigma} \log ^{\tau}|m|}, \quad \beta=\frac{\sigma}{2}
$$

and $s=\alpha$, we have the following lemma.

LEMMA 4. Suppose $\alpha>-1, \sigma \geqslant 0, \alpha+\sigma<(N-1) / 2$ and $\tau$ is a nonnegative number. Then we have

$$
\frac{1}{T} \int_{0}^{T}\left|\sum_{1<|m|^{2}<t}\left(1-\frac{|m|^{2}}{t}\right)^{\alpha} \frac{e^{2 \pi i m x}}{|m|^{\sigma} \log ^{\tau}|m|}\right|^{2} d t=\Omega\left(T^{(N-1) / 2-\alpha-\sigma} \log ^{-2 \tau} T\right)
$$

for every $x$.

3. Proof of theorem. Now let $\sigma$ be equal to $N / p^{\prime}, \tau$ larger than $1 / p$ and $f_{\sigma \tau}$ the function such that

$$
\hat{f}_{\sigma \tau}(m)=\frac{1}{|m|^{\sigma} \log ^{\tau}|m|}, \quad|m|>1 .
$$

Then, from Wainger's theorem [8, Theorem 7] the function $f_{\sigma \tau}$ belongs to $L^{p}\left(T^{N}\right)$ and from Lemma 4 we have

$$
\begin{aligned}
\frac{1}{T} \int_{0}^{T}\left|S_{t}^{\alpha}\left(x, f_{\sigma \tau}\right)\right|^{2} d t & =\frac{1}{T} \int_{0}^{T}\left|\sum_{1<|m|^{2}<t}\left(1-\frac{|m|^{2}}{t}\right)^{\alpha} \frac{e^{2 \pi i m x}}{|m|^{\sigma} \log ^{\tau}|m|}\right|^{2} d t \\
& =\Omega\left(T^{(N-1) / 2-\alpha-N / p^{\prime}} \log ^{-2 \tau} T\right)
\end{aligned}
$$

for every $x$. This completes the proof of theorem.

ACKNOWLEDGMENT. The author is grateful to the referee for correcting the proof of Lemma 2 of the original manuscript.

\section{REFERENCES}

1. Sh. A. Alimov and V. A. Il'in, Conditions for the convergence of expansions corresponding to self-adjoint extensions of elliptic operators. I, Differential Equations 7 (1971), 516-543.

2. Sh. A. Alimov, V. A. Il'in and E. M. Nikishin, Convergence problems of multiple trigonometric series and spectral decompositions. I, Russian Math. Surveys 31 (1976), 29-86.

3. K. I. Babenko, On summability and convergence of the eigenfunction expansions of a differential operator, Math. USSR-Sb. 20 (1973), 157-211.

4. S. Kuratsubo, On summability by the Riesz means of some trigonometric series, Sci. Rep. Hirosaki Univ. 28 (1981), 5-8.

5. B. Novak, Mean value theorems in the theory of lattice point with weight. II, Comment. Math. Univ. Carolin. 11 (1970), 53-81.

6. E. M. Stein, Localization and summability of multiple Fourier series, Acta. Math. 100 (1958), 93-147.

7. E. M. Stein and G. Weiss, Introduction to Fourier analysis on Euclidean spaces, Princeton Univ. Press, Princeton, N. J., 1971.

8. S. Wainger, Special trigonometric series in k-dimensions, Mem. Amer. Math. Soc. No. 59 (1965).

Department of Mathematics, Hirosaki University, Hirosaki, Aomori 036, Japan 